\title{
Mitoxantrone induces apoptosis in osteosarcoma cells through regulation of the Akt/FOXO3 pathway
}

\author{
SEE-HYOUNG PARK ${ }^{1 *}$, JONGSUNG LEE $^{2 *}$, MI-AE KANG $^{3 *}, \mathrm{KYU}^{*}$ YUN JANG ${ }^{4}$ and JUNG RYUL KIM ${ }^{5}$ \\ ${ }^{1}$ Department of Bio and Chemical Engineering, Hongik University, Sejong, Chungcheong 30016; \\ ${ }^{2}$ Department of Genetic Engineering, Sungkyunkwan University, Suwon, Gyeonggi 16419; ${ }^{3}$ Department of \\ Life Science, Gachon University, Seongnam, Gyeonggi 13120; Departments of ${ }^{4}$ Pathology and ${ }^{5}$ Orthopedic Surgery, \\ Chonbuk National University Medical School, Research Institute of Clinical Medicine of Chonbuk National \\ University-Biomedical Research Institute of Chonbuk National University Hospital and Research \\ Institute for Endocrine Sciences, Jeonju, Jeollabuk 54896, Republic of Korea
}

Received October 5, 2017; Accepted March 9, 2018

DOI: $10.3892 / \mathrm{ol} .2018 .8547$

\begin{abstract}
The outcome of chemotherapy for osteosarcoma have improved during the past decade and more patients have access to combination chemotherapy, but there has been no significant clinical progress in the patient survival rate. Recently, forkhead-box O3 (FOXO3) was identified as a pivotal transcription factor responsible for the transcriptional regulation of genes associated with suppression of cancer. The purpose of the present study was to screen small chemicals activating FOXO3 and elucidate their underlying mechanism. Using a drug discovery platform based on the phosphorylation status of FOXO3 in osteosarcoma cells, mitoxantrone (MTZ), a type of DNA-damaging agent, was selected as a possible FOXO3 activator from the food and drug administration-approved drug library. MTZ treatments significantly inhibited the phosphorylation level of Akt-pS473 and caused nuclear localization of FOXO3 in osteosarcoma cells. MTZ treatment inhibited proliferation in osteosarcoma cells in vitro, whereas silencing
\end{abstract}

Correspondence to: Professor Jung Ryul Kim, Department of Orthopedic Surgery, Chonbuk National University Medical School, Research Institute of Clinical Medicine of Chonbuk National University-Biomedical Research Institute of Chonbuk National University Hospital and Research Institute for Endocrine Sciences, 567 Baekje-daero, Jeonju, Jeollabuk 54896, Republic of Korea E-mail: jrkeem@jbnu.ac.kr

Professor Kyu Yun Jang, Department of Pathology, Chonbuk National University Medical School, Research Institute of Clinical Medicine of Chonbuk National University-Biomedical Research Institute of Chonbuk National University Hospital and Research Institute for Endocrine Sciences, 567 Baekje-daero, Jeonju, Jeollabuk 54896, Republic of Korea

E-mail: kyjang@jbnu.ac.kr

${ }^{*}$ Contributed equally

Key words: mitoxantrone, apoptosis, osteosarcoma, Akt, forkhead box $\mathrm{O} 3$
FOXO3 potently attenuates MTZ-mediated apoptosis in osteosarcoma cells. Taken together, the results indicated that MTZ induces apoptosis in osteosarcoma cells through an Akt/FOXO3-dependent mechanism.

\section{Introduction}

In 2014, osteosarcoma was among the 10 most common cancers in children and teenagers in the USA; about half of the 800 patients with osteosarcoma diagnosed annually are children and teenagers (1). The long bones in the legs and arms are the most common sites of osteosarcoma (2). Metastasis of osteosarcoma is determined in $>30 \%$ of patients who received only surgical treatment (3). The lung is the most common metastasis organ for osteosarcoma (3). According to a recent study, the probability of successful treatment for non-metastatic osteosarcoma is around 70\%; however, following metastasis of osteosarcoma cells to other organs, the treatment success rate may decrease by half, down to as low as $20 \%$ (4). The survival rate of patients with osteosarcoma treated with the combined chemotherapy of cisplatin, doxorubicin and high-dose methotrexate is $\sim 70 \%$, demonstrating the potential of this chemotherapeutic regimen (5). However, there is still room for improvement, and the side effects of current chemotherapy should be reduced; therefore, research focusing on safer and more effective therapeutic options is urgently required.

Forkhead-box O3 (FOXO3) is a isomer of the FOX gene family (6). FOXO3 is a tumor-suppressive transcriptional factor that controls various cellular processes, including cell cycle arrest, apoptosis and tumor suppression, in various kinds of cancer $(7,8)$. FOXO3 is regulated via phosphorylation of specific serine/threonine residues by oncogenic kinases in cancer cells (9). It has been reported that Akt phosphorylates specific serine/threonine residues (Thr32, Ser253 and Ser315) on FOXO3, leading to the degradation of FOXO3 following translocation from the nucleus to the cytoplasm (10). Translocation of FOXO3 into the cytoplasm inhibits its tumor suppressive transcriptional activity, which 
results in tumor development and progression $(11,12)$. Notably, numerous clinical studies using tissue microarrays (TMAs) to characterize the association between the nuclear localization or expression levels of FOXO3 and the survival rates of patients with ovarian cancer have revealed that FOXO3 is a good prognostic biomarker $(13,14)$; thus, FOXO3 activation in cancer cells may provide a promising strategy for developing anti-cancer therapeutic drugs.

Mitoxantrone (MTZ; Fig. 1), a synthetic anti-tumor derivative of anthracycline antibiotics, has been used largely for the treatment of tumor types, including leukemia, lymphoma and prostate cancer, as well as multiple sclerosis (15-18). MTZ is known to inhibit topoisomerase II, which prevents the rejoining of DNA strands during the DNA replication step, causing DNA damage by DNA double-strand breakage (19). Consequently, MTZ affects the cell cycle and induces apoptosis in cancer cells (20). In the present study, the potential of MTZ as an anti-cancer therapeutic option for treating osteosarcoma was demonstrated by indicating the underlying mechanism.

To develop a novel therapy against osteosarcoma, MTZ was selected as a FOXO3 activator in osteosarcoma cells by applying drug repositioning and investigating the anti-cancer activity and underlying mechanisms of MTZ. Drug repositioning refers to the application of previously clinically-used drugs against a specific disease, then used for another disease by evaluating novel drug activity, which saves time and cost for drug development (21). Based on the data, it was indicated that MTZ treatment in osteosarcoma cells induces apoptosis through FOXO3 upregulation, which increases pro-apoptotic genes but decreases cell survival genes. FOXO3 is a promising candidate for the development of osteosarcoma therapy, as these therapies may sensitize osteosarcoma cells to FOXO3-mediated apoptosis and suppress tumorigenesis.

\section{Materials and methods}

Cell culture. U2OS (p53 wild) and MG63 (p53 null) cells (American Type Culture Collection, Manassas, VA, USA) were maintained in Dulbecco's modified Eagle's media with $10 \%$ fetal bovine serum (FBS) and 1\% streptomycin/penicillin (Gibco; Thermo Fisher Scientific, Inc., Waltham, MA, USA) at $37^{\circ} \mathrm{C}$ in a humidified incubator containing $5 \% \mathrm{CO}_{2}$ in air. Both cell lines were used at passages 4-10 for all experiments.

Chemical reagents and antibodies. Mouse anti- $\beta$-actin antibody (cat. no. A5441), MTZ (cat. no. M6545) and the following chemicals and solvents, dimethyl sulfoxide (DMSO), glycerol, glycine, sodium chloride, Trizma base and Tween20, were purchased from Sigma-Aldrich (Merck KGaA, Darmstadt, Germany). Mouse anti-poly(ADP-ribose) polymerase(PARP)1 (cat. no. sc-8007), rabbit anti-FOXO3 (cat. no. sc-11351), mouse anti-Lamin B1 (cat. no. sc-56145) and mouse anti-GAPDH antibodies (cat. no. sc-32233) were purchased from Santa Cruz Biotechnology, Inc. (Dallas, TX, USA). Rabbit anti-pAkt (cat. no. 9271), rabbit anti-Akt (cat. no. 4691), rabbit anti-cleaved PARP1 (cat. no. 9541), rabbit anti-cleaved Caspase-3 (cat. no. 9664), rabbit anti-Bax (cat. no. 5023), rabbit anti-Bim (cat. no. 2933), rabbit anti-Bcl2 (cat. no. 2872) and rabbit anti-p27 antibodies (cat. no. 3686) were purchased from Cell Signaling Technology, Inc. (Danvers, MA, USA).
Goat anti-rabbit (cat. no. 111-035-003) and goat anti-mouse (cat. no. 115-035-003) horseradish peroxidase-conjugated IgG were obtained from Jackson ImmunoResearch Laboratories, Inc. (West Grove, PA, USA). Enhanced chemiluminescence (ECL) reagents were obtained from GenDEPOT (Barker, TX, USA).

WST-1 assay. Cells $\left(1 \times 10^{3}\right)$ were seeded in each well of a 96-well plate and incubated for $18 \mathrm{~h}$ at $37^{\circ} \mathrm{C}$ in a humidified incubator containing $5 \% \mathrm{CO}_{2}$ in air. Following incubation, cells were treated with DMSO $(0.1 \%)$ as a control vehicle and $0,0.1,0.2,0.5,0.8$, and $1 \mu \mathrm{M}$ indicated concentration of MTZ for $72 \mathrm{~h}$ at $37^{\circ} \mathrm{C}$. Following this, $20 \mu \mathrm{l}$ WST-1 solution (DoGenBio, Seoul, Korea) was added to each well for $4 \mathrm{~h}$. The visible absorbance at $460 \mathrm{~nm}$ for each well was then quantified using a microplate reader. The assay was repeated 3 times.

Colony formation assay. Cells $\left(0.5 \times 10^{3}\right)$ were seeded in $6 \mathrm{~cm}$ dishes and incubated for $18 \mathrm{~h}$ at $37^{\circ} \mathrm{C}$ in a humidified incubator containing $5 \% \mathrm{CO}_{2}$ in air. Following incubation, cells were treated with DMSO $(0.1 \%)$ as a control vehicle or the indicated concentration of MTZ for 7 days. The colonies were washed twice with PBS, fixed with $3.7 \%$ paraformaldehyde and stained with $1 \%$ crystal violet solution in distilled water at room temperature for $15 \mathrm{~min}$. Images were captured using the Chemi-doc detection system (Bio-Rad Laboratoried, Hercules, CA, USA). The assay was repeated 3 times.

Western blot analysis. Cells were washed 3 times with PBS and lysed in lysis buffer $(50 \mathrm{mM}$ Tris- $\mathrm{HCl}, 150 \mathrm{mM} \mathrm{NaCl}$, 2 mM EDTA, $1 \%$ Triton X-100 and 0.1\% SDS; pH 8.0) with protease and phosphatase inhibitors (Sigma-Aldrich; Merck KGaA, Darmstadt, Germany). Cell lysates were centrifuged $\left(10,000 \mathrm{x} \mathrm{g}\right.$ at $4^{\circ} \mathrm{C}$ for $\left.10 \mathrm{~min}\right)$. The protein concentration was measured using a Bradford protein determination assay (Bio-Rad Laboratories) and $20 \mu \mathrm{g}$ protein was loaded per the lane. Proteins were separated on $10 \%$ SDS-PAGE gels and blotted onto nitrocellulose membranes (Bio-Rad Laboratories, Inc., Hercules, CA, USA). The membranes were blocked in 3\% non-fat dry milk for $1 \mathrm{~h}$ at room temperature and probed with primary antibodies for FOXO3, pAkt, Akt, p27, LaminB1, GAPDH, cleaved caspase3, Bim (EL), Bax, $\mathrm{Bcl} 2$, and $\beta$-actin. All antibodies were diluted at 1:1,000 and incubated for $1 \mathrm{~h}$ at room temperature. Membranes were then probed with HRP-tagged goat anti-mouse or anti-rabbit IgG antibodies, diluted at 1:15,000 and 1:5,000 respectively, for $1 \mathrm{~h}$ at room temperature. Chemiluminescence was detected using ECL.

Cell-based enzyme-linked immunosorbent assay (ELISA). $\mathrm{U} 2 \mathrm{OS}$ cells $\left[2 \times 10^{4} /\right.$ well in $100 \mu \mathrm{l}$ DMEM (Gibco; Thermo Fisher Scientific, Inc.)] were seeded into 96-well plates and incubated at $37^{\circ} \mathrm{C}$ for $18 \mathrm{~h}$ in a $\mathrm{CO}_{2}$ incubator. Cells in each well were treated separately with DMSO (negative control), LY294002 (Sigma-Aldrich; Merck KGaA) or Wortmannin (positive control; Sigma-Aldrich; Merck KGaA), and small-molecule compounds (20 $\mu \mathrm{M} / \mathrm{ml}$ final conc.; http://www.enzolifesciences. com/BML-2843/screen-well-fda-approved-drug-library-v2/) from the FDA-Approved Drug Library (Enzo Life Sciences, Inc., Farmingdale, NY, USA) (totaling 640 drugs) at $37^{\circ} \mathrm{C}$ and 
$5 \% \mathrm{CO}_{2}$ for $24 \mathrm{~h}$. Then, cells were fixed and quenched by adding $100 \mu 14 \%$ formaldehyde (in PBS) at room temperature for $15 \mathrm{~min}$ and $100 \mu 10.6 \% \mathrm{H}_{2} \mathrm{O}_{2}$ (in PBS) at room temperature for $15 \mathrm{~min}$. To determine the level of the phosphorylated-FOXO3-S318/321 in these cells, ELISA was performed by treating cells with $100 \mu \mathrm{l}$ blocking buffer [5\% bovine serum albumin (BSA) in Tris-buffered saline with $0.1 \%$ Tween-20 (TBST)] at room temperature for $1 \mathrm{~h}$. Subsequent to washing the cells with TBST 3 times, cells were treated with $100 \mu 1$ primary antibody against phospho-FOXO3-S318/321 (9465; 1:250 dilution in TBST containing 2\% BSA; Cell Signaling Technology, Inc.) at $4^{\circ} \mathrm{C}$ overnight. Subsequent to washing, cells with TBST three times were treated with $100 \mu \mathrm{l}$ anti-rabbit IgG horseradish peroxidase-conjugated secondary antibody (1:1,000 dilution in TBST containing 2\% BSA; Jackson ImmunoResearch Laboratories, Inc.) at room temperature for $2 \mathrm{~h}$. Following washing cells with TBST 3 times, cells were treated with $100 \mu 1$ 3,3',5,5'-tetramethylbenzidine peroxidase substrate (Sigma-Aldrich; Merck $\mathrm{KGaA}$ ) for $15 \mathrm{~min}$ at room temperature, followed by adding $50 \mu \mathrm{l}$ ELISA stop solution $\left(2 \mathrm{~N} \mathrm{H}_{2} \mathrm{SO}_{4}\right.$; Sigma-Aldrich; Merck $\mathrm{KGaA}$ ). The optical density of each well was measured by reading the microplate using a microplate reader (Molecular Devices, LLC, Sunnyvale, CA, USA) at $450 \mathrm{~nm}$.

Cytoplasmic and nuclear protein fractionation. Cells were washed 3 times with PBS and lysed in cytoplasmic fractional buffer [10 mM 4-(2-hydroxyethyl)-1-piperazineethanesulphonic acid (HEPES), pH 8.0, $50 \mathrm{mM} \mathrm{NaCl}, 500 \mathrm{mM}$ sucrose, $1 \mathrm{mM}$ EDTA, $0.5 \mathrm{mM}$ spermidine, $0.15 \mathrm{mM}$ spermine, $0.2 \%$ Triton $\mathrm{X}-100,1 \mathrm{mM}$ dithiothreitol, $2 \mu \mathrm{M}$ phenylmethylsulfonyl fluoride and $0.15 \mathrm{U} / \mathrm{ml}$ aprotinin] at $4^{\circ} \mathrm{C}$ for $30 \mathrm{~min}$. Cell lysates were centrifuged $(10,000 \mathrm{x} \mathrm{g}$ at $4^{\circ} \mathrm{C}$ for $30 \mathrm{~min}$ ) and the supernatant was collected for the cytoplasmic fraction. The pellet was washed twice with the washing buffer (10 mM HEPES pH 8.0, $50 \mathrm{mM}$ $\mathrm{NaCl}, 25 \%$ glycerol, $0.1 \mathrm{mM}$ EDTA, $0.5 \mathrm{mM}$ spermidine and $0.15 \mathrm{mM}$ spermine) and lysed with nuclear fractional buffer (10 mM HEPES pH 8, $350 \mathrm{mM} \mathrm{NaCl,} \mathrm{25 \%} \mathrm{glycerol,}$ $0.1 \mathrm{mM}$ EDTA, $0.5 \mathrm{mM}$ spermidine and $0.15 \mathrm{mM}$ spermine) at $4^{\circ} \mathrm{C}$ for $30 \mathrm{~min}$. Lysates were centrifuged $(10,000 \mathrm{x} \mathrm{g}$ at $4^{\circ} \mathrm{C}$ for $30 \mathrm{~min}$ ) and the supernatant was collected for the nuclear fraction. All of buffers used in this experiment were prepared in the Park laboratory (Sejong, Korea), based on the previous report (12).

Immunofluorescence analysis. Cells $\left(5 \times 10^{5}\right)$ were seeded in $6 \mathrm{~cm}$ dishes and incubated for $18 \mathrm{~h}$ at $37^{\circ} \mathrm{C}$ in a humidified incubator containing $5 \% \mathrm{CO}_{2}$ in air. Following incubation, cells were treated with DMSO $(0.1 \%)$ as a control vehicle and $1 \mu \mathrm{M} \mathrm{MTZ}$ for $2 \mathrm{~h}$ sat $37^{\circ} \mathrm{C}$. Cells were fixed with $4 \%$ paraformaldehyde solution for $15 \mathrm{~min}$ at room temperature, permeabilized with Triton X-100 (0.2\%), blocked with bovine serum albumin and incubated with the primary antibody against FOXO3 (diluted, 1,500) at room temperature for $1 \mathrm{~h}$, followed by Alexa 594-conjugated anti-mouse secondary antibody (cat. no. A-11037; Invitrogen; Thermo Fisher Scientific, Inc., Waltham, MA, USA) a room temperature for $1 \mathrm{~h}$. Following counterstaining with DAPI at room temperature for $1 \mathrm{~h}$, fluorescence images were captured with a Zeiss LSM510 confocal microscope at magnification, $\mathrm{x} 100$.
Terminal deoxynucleotidyl-transferase-mediated dUTP nick end labeling (TUNEL) assay. Cells $\left(5 \times 10^{5}\right)$ were seeded in $6 \mathrm{~cm}$ dishes and incubated for $18 \mathrm{~h}$ at $37^{\circ} \mathrm{C}$ in a humidified incubator containing $5 \% \mathrm{CO}_{2}$ in air. Following incubation, cells were treated with DMSO $(0.1 \%)$ as a control vehicle and 0.5 and $1 \mu \mathrm{M}$ MTZ for $2 \mathrm{~h}$. Cells were fixed with $4 \%$ paraformaldehyde solution for $15 \mathrm{~min}$ at room temperature and permeabilized with Triton X-100 (0.2\%). Apoptosis was determined by enzymatic labeling of DNA strand breaks with a TUNEL assay kit (the DeadEnd Fluorometric TUNEL System; Promega Corporation, Madison, WI, USA), according to the manufacturer's procedures. Fluoroshield Mounting Medium with DAPI (Abcam, Cambridge, UK) was used, and 3 fields of view were imaged using the Olympus CKX53 light microscope (Olympus Corporation, Tokyo, Japan; magnification, x100).

Annexin V staining analysis. Cells $\left(5 \times 10^{5}\right)$ were seeded in $6-\mathrm{cm}$ dishes and incubated for $18 \mathrm{~h}$ at $37^{\circ} \mathrm{C}$ in a humidified incubator containing $5 \% \mathrm{CO}_{2}$ in air. Following incubation, cells were treated with DMSO $(0.1 \%)$ as control vehicle and 0.5 and $1 \mu \mathrm{M}$ MTZ for $2 \mathrm{~h}$. Cells were washed 3 times with PBS, trypsinized with Gibco $^{\text {TM }}$ Trypsin-EDTA (0.25\%) with Phenol red (Gibco; Thermo Fisher Scientific, Inc.) and resuspended in binding buffer from the Annexin V Apoptosis Detection kit I (BD Pharmingen; BD Biosciences). Cells were analyzed using FACSCalibur (BD Biosciences, Franklin Lakes, NJ, USA) and the data were analyzed by FlowJo (version 10; De Novo Software, Ashland, Oregon, USA). A total of 10,000 events were collected in each run. The percentage of cells that underwent apoptosis was determined using the fluorescein isothiocyanate with propidium iodide from the Annexin V Apoptosis Detection kit I, according to the manufacturer's procedures.

siRNA transfection. Cells $\left(5 \times 10^{5}\right)$ were seeded in 6 -cm dishes and incubated for $18 \mathrm{~h}$ at $371 \mathrm{~s}$ that humidified incubator containing $5 \% \mathrm{CO}_{2}$ in air. Following incubation, cells were transfected with control (GE Healthcare Dharmacon, Inc., Lafayette, CO, USA) or FOXO3 siRNA (GE Healthcare Dharmacon, Inc.) using the ratio of $1 \mathrm{nM}$ siRNA: $3 \mu \mathrm{l}$ Dharmafect (GE Healthcare Dharmacon, Inc.) in $300 \mu \mathrm{l}$ serum free media for $6 \mathrm{~h}$ at $37^{\circ} \mathrm{C}$ in a humidified incubator containing $5 \% \mathrm{CO}_{2}$ in air. Following this, cell culture media was replaced with the fresh media containing 10\% FBS and cells were incubated for $18 \mathrm{~h}$.

Statistical analysis. Data are expressed as the mean \pm standard error of three independent experiments. Differences between groups were analyzed with one-way analysis of variance with Tukey's post-hoc test when the variances were equal with SPSS software (version 19.0; IBM Corp., Armonk, NY, USA). All statistical tests were two-sided. $\mathrm{P}<0.05$ was considered to indicate a statistically significant difference.

\section{Results}

MTZ induces nuclear localization and activation of $\mathrm{FOXO3}$ in osteosarcoma cells. To identify small molecules that can activate FOXO3 in U2OS cells, a osteosarcoma cell line, a 


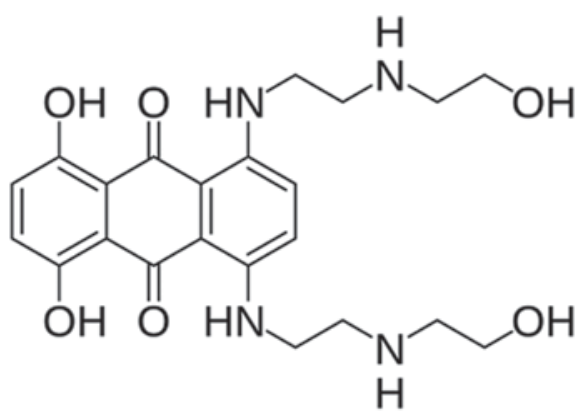

Figure 1. Chemical structure of MTZ. MTZ is an anthraquinone-based anti-cancer agent. MTZ, mitoxantrone.

cell-based enzymatic ELISA was performed to screen small molecules that may significantly reduce the phosphorylation of Ser318/321 in FOXO3 (pS318/321 FOXO3), which is usually located in the cytoplasm of cells (10). Various FOXO3 phosphorylation sites have been identified by a number of kinases, and these serve a key role in regulating the cellular location and transcriptional activity of FOXO3. Among these, Ser318 and Ser322 have been investigated by Rena et al (22). They indicated that the phosphorylation of Ser318 by Akt induces the subsequent phosphorylation of Ser322 by casein kinase 1 , which is critical for translocation of FOXO3 from the nucleus into the cytosol. In previous studies $(11,12,23)$, attempts were made to develop a novel cell-based ELISA assay system using a phospho-FOXO3 antibody to screen small molecules causing nuclear localization of FOXO3. Following testing various commercially available phospho-FOXO3 antibodies including pThr32 and pSer253, which are possible Akt-phosphorylation sites identified by Brunet et al (10), pSer318/321 FOXO3 antibody was selected as the best antibody for the cell-based ELISA system, due to a larger difference in the readout value being detected between the positive controls (Wortmannin and LY294002) and the negative control. Following screening small molecule drugs from a commercially available food and drug administration (FDA)-approved drug library (SCREEN-WELL ${ }^{\circledR}$ FDA approved drug library V2: BML-2843-0100; Enzo Life Sciences, Inc.), MTZ was focused on (Fig. 1), which indicated a decreased level (>50\%) of pS318/321 FOXO3, compared with the DMSO vehicle control in U2OS cells (data not shown).

To demonstrate the mechanism by which MTZ treatment decreases pS318/321 FOXO3 in U2OS cells, nuclear fractional western blot analyses with lysates from U2OS cells previously treated with various doses of MTZ were performed. As depicted in Fig. 2A, MTZ treatment reduced the expression of Akt-pS473 (pS473 Akt) in the cytoplasm, in a dose-dependent manner. In addition, the expression of FOXO3 in the cytoplasm was decreased by MTZ treatment, whilst the expression of FOXO3 in the nucleus was significantly increased by MTZ treatment, in a dose-dependent manner. The expression of the cytoplasmic and nuclear p27 protein was significantly increased by MTZ treatment, in a dose-dependent manner. Additionally, it was confirmed that FOXO3 translocates into the nucleus following MTZ treatment, by carrying out confocal microscopy analysis (Fig. 2B). These results indicated that MTZ may downregulate pS473 Akt, leading to the translocation and activation of the $\mathrm{FOXO} 3$ protein from the cytoplasm to the nucleus in osteosarcoma cells.

MTZ suppresses cell survival and promotes apoptosis in osteosarcoma cells. To investigate the anti-cancer activity of MTZ against osteosarcoma cells, U2OS and MG63 osteosarcoma cells were treated with MTZ and growth rate of the cells was measured using the WST-1 and colony formation assays. Cell viability was determined using WST-1 assay, which involved incubating the U2OS and MG63 cells with MTZ $(0,0.1,0.2,0.5,0.8$ and 1.0 for 72 h. As depicted in Fig. 3A, MTZ had an inhibitory effect on osteosarcoma cell growth, in a dose-dependent manner. Notably, treatment with 0.5, 0.8 and $1 \mu \mathrm{M}$ MTZ resulted in a statistically significant $(\mathrm{P}<0.05)$ inhibition of cell viability; therefore, based on the data on cell viability/cytotoxicity, it may be concluded that $\geq 0.5 \mu \mathrm{M}$ MTZ is the optimal dose for the subsequent experiments. In addition, clonogenic assay results demonstrated that MTZ treatment markedly suppressed the colony-forming ability of osteosarcoma cells (Fig. 3B). Taken together, these results indicated that MTZ displays a potent inhibitory effect on the cell survival/proliferation of osteosarcoma cells. To examine the effect of MTZ on apoptosis in osteosarcoma cells, western blot analyses, TUNEL assays and Annexin V staining analysis were performed. MTZ treatment increased the cleavage of Caspase-3 and PARP1. MTZ treatment also increased the expression of Bax and Bim extra-large (EL) in osteosarcoma cells; however, MTZ treatment decreased the expression of Bcl-2 (Fig. 3C). These results indicated that the mechanism underlying MTZ and its apoptotic activity may be through a Caspase-3-mediated mechanism in osteosarcoma cells, upregulation of the pro-apoptotic Bax and Bim and downregulation of the anti-apoptotic $\mathrm{Bcl} 2$. In addition, compared with the DMSO control, MTZ treatment of osteosarcoma cells resulted in an increase in the amount of Annexin V stained cells as well as TUNEL-positive cells, both of which are typical markers of apoptosis (Fig. 4A and B). Thus, the present results indicated that MTZ treatment may induce cellular apoptosis in osteosarcoma cells.

Knockdown of FOXO3 decreases MTZ-mediated apoptosis in osteosarcoma cells. To examine the role of FOXO3 in MTZ-induced apoptosis in osteosarcoma cells, FOXO3 expression was knocked down in U2OS cells by transfecting siRNA against FOXO3 or control siRNA. Additionally, western blot analysis of PARP1, Caspase-3, Bax, Bim EL, Bcl2 and FOXO3 was performed. As depicted in Fig. 5, FOXO3 knockdown markedly attenuated Caspase-3 and PARP1 cleavage and reduced the expression levels of Bax and the Bim EL isoform, compared with the control siRNA. However, MTZ treatment decreased the reduced the expression levels of Bcl-2. Thus, the present results indicated that FOXO3 may serve a key role in MTZ-mediated apoptosis in U2OS cells.

\section{Discussion}

Osteosarcoma was demonstrated in 2014 to be the most common malignant tumor type of childhood (1) The incidence of osteosarcoma in young children is $>10 \%$ (24). There are three types of osteosarcoma, low, middle and high grade 
A

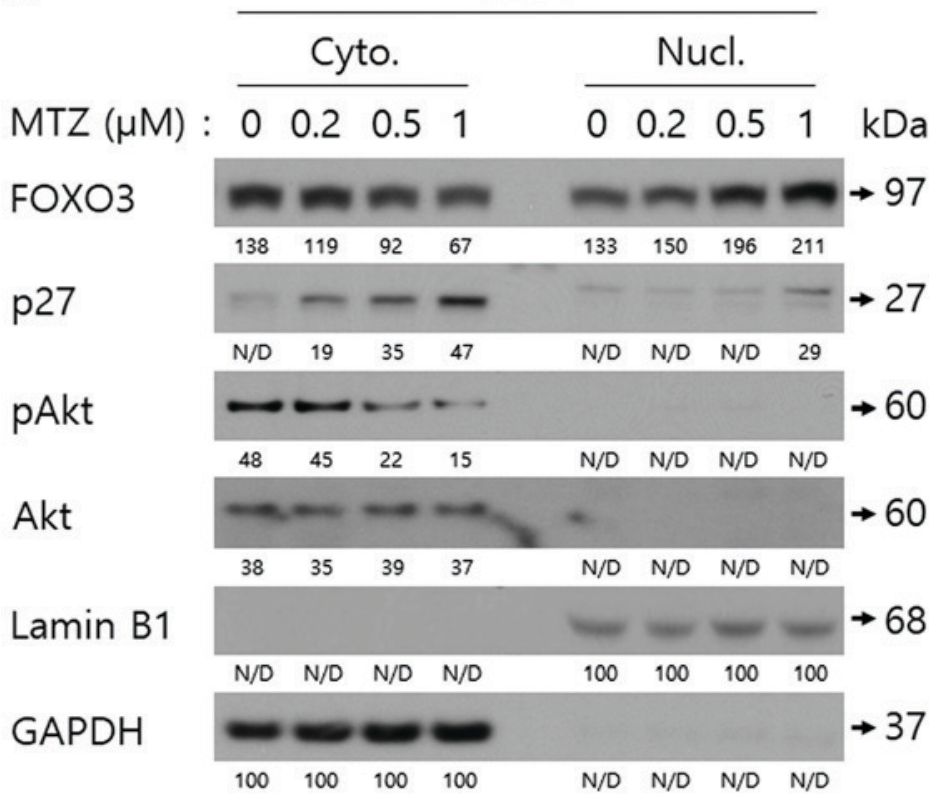

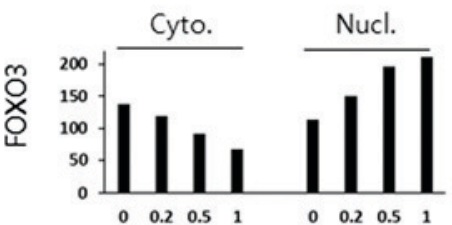
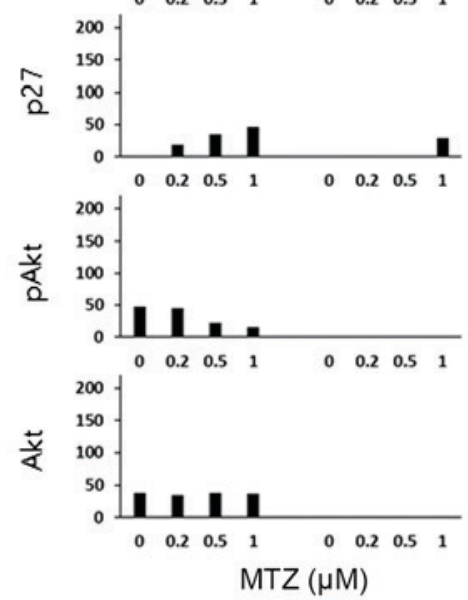

B U2OS
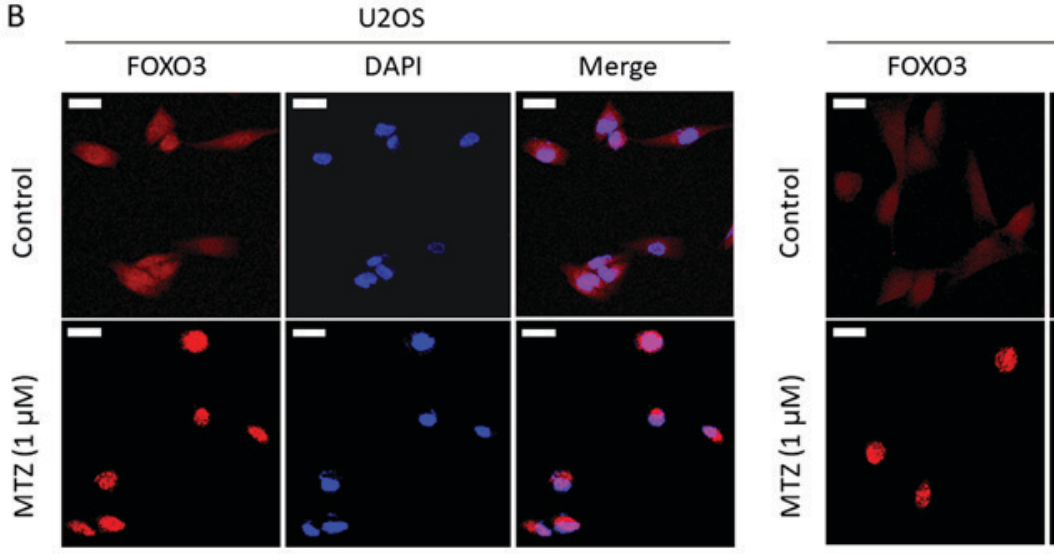

MG63

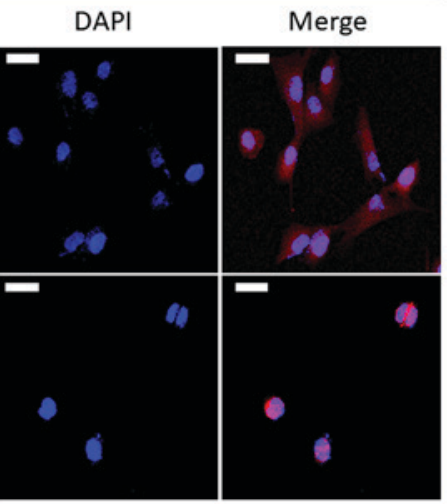

Figure 2. Inhibition of pS473 Akt and translocation of FOXO3 protein from the cytoplasm to the nucleus in osteosarcoma cells treated with MTZ. (A) Nuclear fraction western blot analysis results of U2OS cells treated with MTZ $(0,0.25,0.5$ and $1 \mu \mathrm{M})$. The levels of the indicated proteins in the cytoplasm and nucleus were analyzed by western blotting using specific antibodies. GAPDH and Lamin B1 were as loading controls. (B) Confocal microscopy results of U2OS and MG63 cells treated with MTZ $(1 \mu \mathrm{M})$ for $2 \mathrm{~h}$. Following counterstaining with DAPI to indicate the nuclei, fluorescence images were captured with a confocal microscope. Scale bar, $10 \mu \mathrm{m}$. Cyto, cytoplasm; nucl, nuclei; FOXO3, forkhead-box O3; MTZ, mitoxantrone.

osteosarcoma (25). In cases of low-grade osteosarcoma, such as parosteal osteosarcoma, patients have a good prognosis, with a $91 \%$ five-year survival rate following surgery alone (26). In middle-grade osteosarcoma, although surgery has success occasionally, the effectiveness of additional chemotherapy has not been clearly demonstrated $(27,28)$. High-grade osteosarcoma is the most common subtype and is responsible for $\sim 80 \%$ of osteosarcomas. The use of combined chemotherapy before or following surgery may improve the survival rate of patients by $>60 \%$, compared with surgery alone (29). Thus, although combined aggressive chemotherapy using usually DNA damaging agent has been developed, $>30 \%$ of patients with osteosarcoma still have metastatic cancer in other tissues and the survival rate with metastases is $<1$ year (3); therefore, more effective therapeutic options are required for the treatment of osteosarcomas.

There are standard combined chemotherapy treatment protocols for osteosarcoma depending on the stage (first and second-line treatment). First line treatment includes the combination of: i) Cisplatin and doxorubicin; ii) cisplatin, doxorubicin and methotrexate; iii) etoposide and ifosfamide; or iv) cisplatin, epirubicin and ifosfamide (30-32). Second line treatment involves the combination or sole use of: i) gemcitabine and docetaxel; ii) etoposide and cyclophosphamide; iii) topotecan and cyclophosphamide; iv) gemcitabine; v) etoposide and ifosfamide; vi) carboplatin, etoposide and ifosfamide; vii) etoposide, ifosfamide and methotrexate; or viii) Samarium-153-ethylene diamine tetramethylene phosphonate (33-40). However, several recent reports have demonstrated that resistance against these chemotherapies requires consideration (41-43). There are several publications dealing with p53 mutations in patients with osteosarcoma (44-46). p53 is a tumor-suppressive transcription factor that mediates apoptosis following DNA damage in cells $(47,48)$; therefore, it is difficult to successfully treat patients with osteosarcoma with p53 mutation by using chemotherapy, which activates p53. However, there are no studies regarding FOXO3 mutations in patients with osteosarcoma; therefore, it is beneficial to 
A

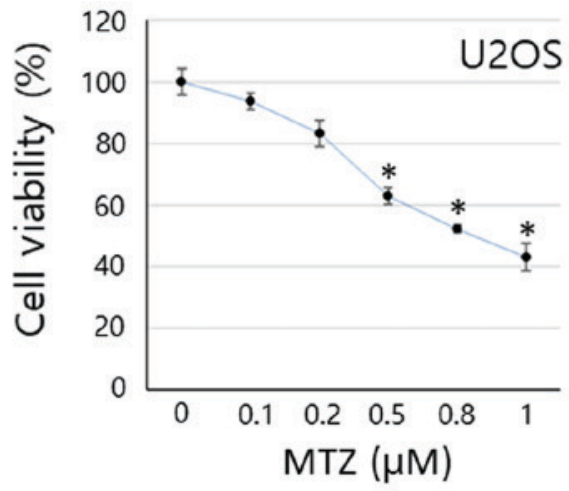

B MTZ ( $\mu M)$ :

U2OS
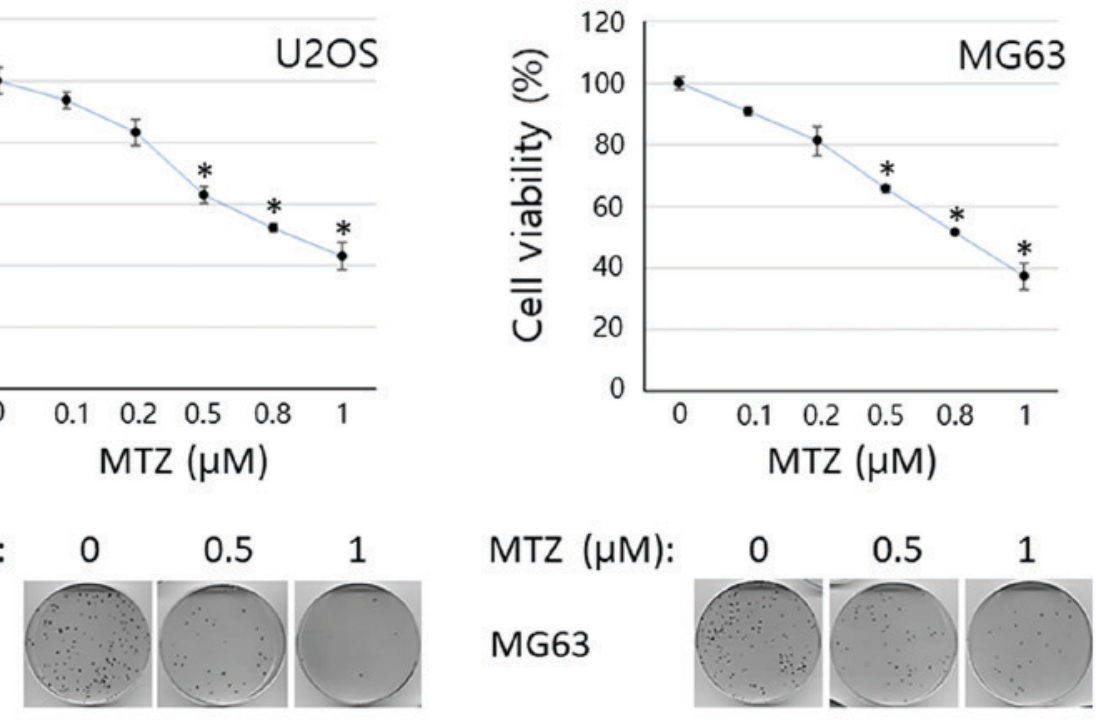

C
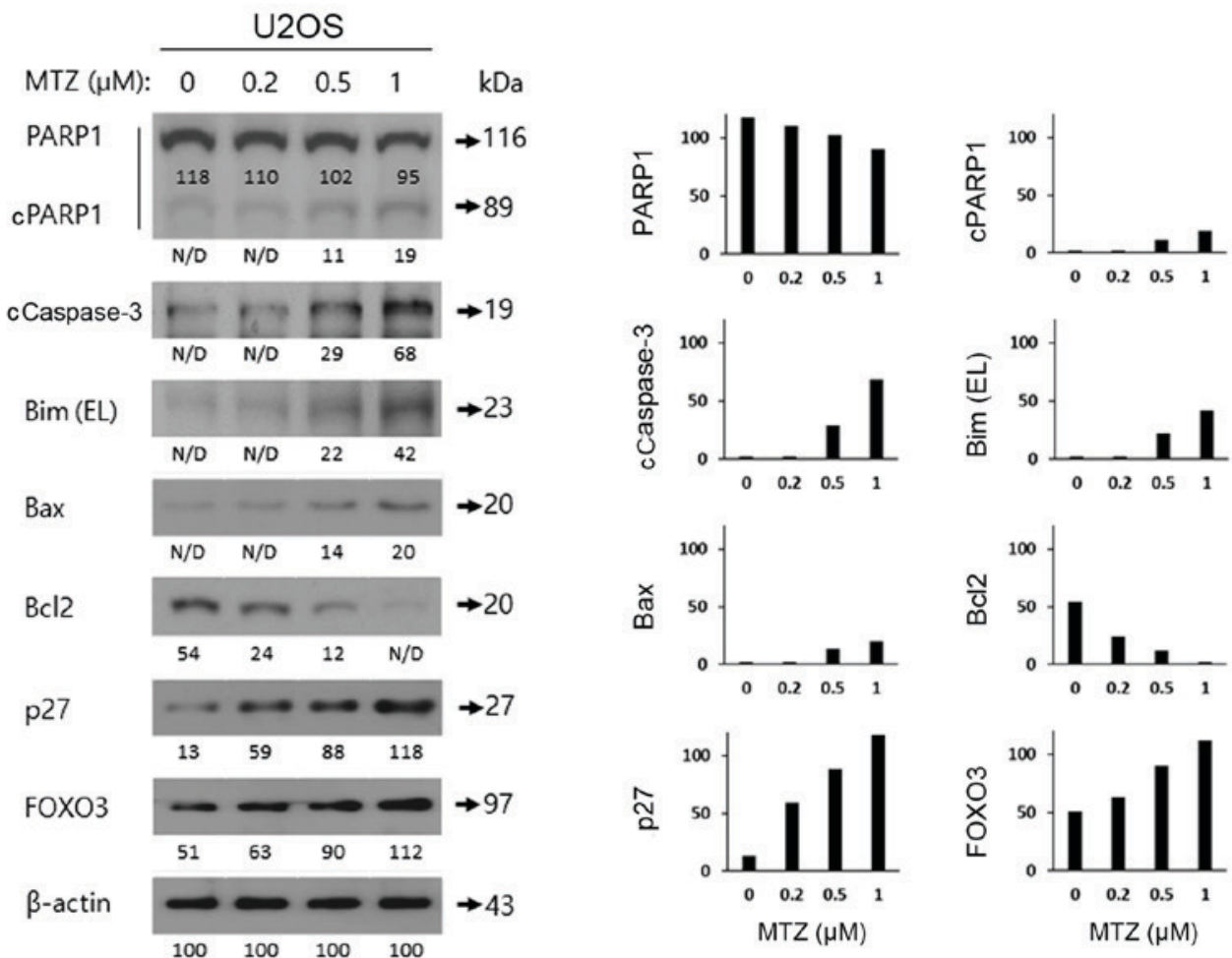

Figure 3. Anti-cancer effect of MTZ against osteosarcoma cells. (A) Dose-dependent effects of MTZ $(0,0.1,0.2,0.5,0.8$ and 1 cancer effect of MTZ against ost following $72 \mathrm{~h}$ incubation. The cell viability was determined by WST-1 assay and the relative cell survival rate was calculated by dividing the optical density of each treatment condition by the optical density of the control (DMSO) treatment. The mean of 3 biological replicates is presented and the error bars represent standard error of the mean. "P<0.05. (B) Dose-dependent effect of MTZ ( 0.5 and $1 \mu \mathrm{M})$ on U2OS and MG63 cells following 7 days. Represented images of 3 scanned images are provided. (C) Apoptosis analysis of U2OS cells treated with MTZ and western blotting results of U2OS cells treated with MTZ $(0,0.2$, 0.5 , and $1 \mu \mathrm{M}$ ) for $48 \mathrm{~h} . \beta$-actin was used for a gel-loading control. Quantitative data are based on the relative ratio of the indicated protein to $\beta$-actin. MTZ, mitoxantrone; EL, extra-large; FOXO3, forkhead-box O3; PARP1, poly(ADP-ribose) polymerase; c, cleaved.

consider novel combination chemotherapies that may activate FOXO3 using MTZ and other FDA-approved drugs (11). Future studies will examine the synergic effect of MTZ with other FOXO3-activating small molecules, with the goal of inhibiting osteosarcoma cell development.

In the present study, the aim was to identify small molecules that activate $\mathrm{FOXO}$, and as a result $\mathrm{FOXO}$-mediated apoptosis, in osteosarcoma cell lines, which can be used as a novel therapeutic target. Previously, it was demonstrated that trifluoperazine and bepridil may promote the translocation of FOXO3 into the nucleus in triple-negative breast cancer (TNBC) cells, leading to suppression of TNBC in vitro and in vivo (11). In the present study, using the same drug discovery platform (cell-based ELISA), based on the phosphorylation status of FOXO3 in U2OS cells, a possible FOXO3 activator from an FDA-approved drug library was screened. The focus was on MTZ, a type of DNA damaging agent, for the present study due to MTZ displaying the 
A

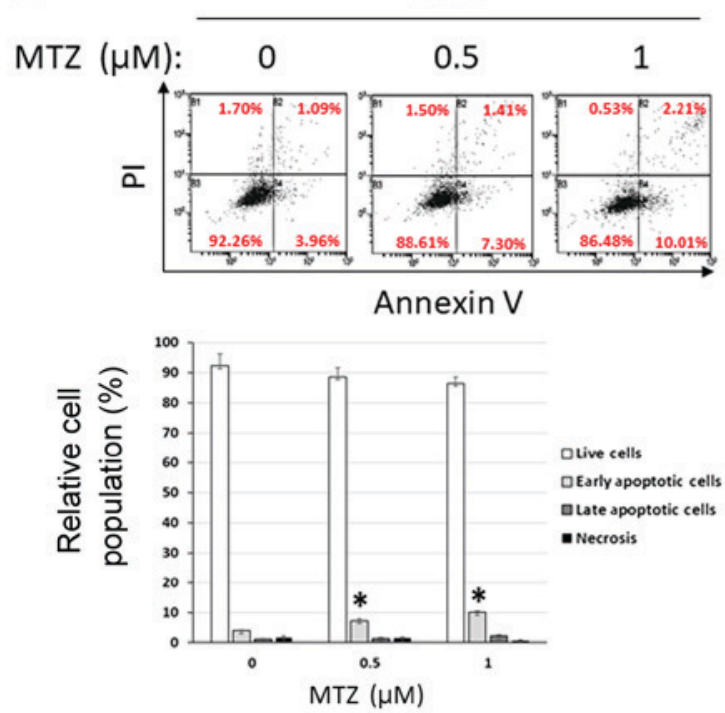

B

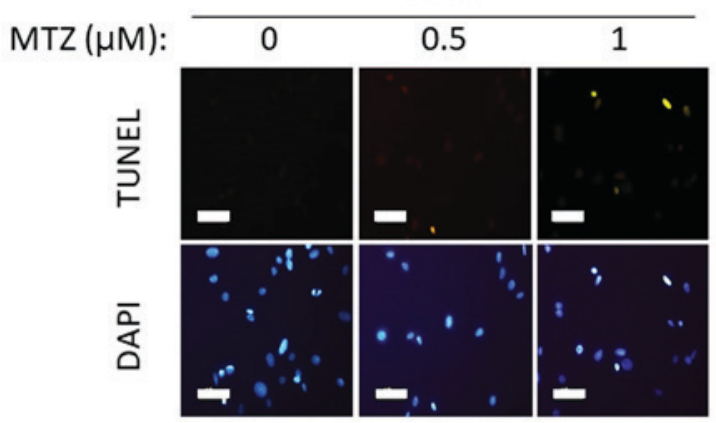

MG63
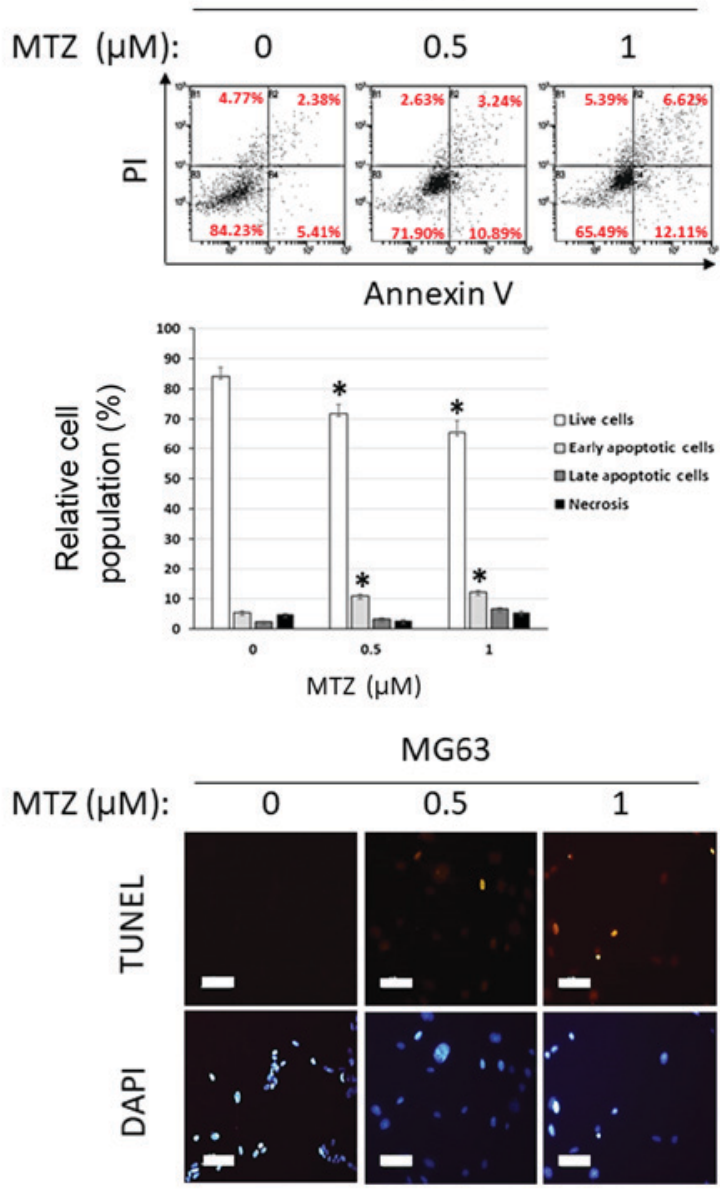

Figure 4. Annexin V staining and TUNEL analysis in osteosarcoma cells treated with MTZ. (A) Annexin V staining results of U2OS and MG63 cells treated with MTZ $(0,0.5$ and $1 \mu \mathrm{M})$ for $2 \mathrm{~h}$. The representative images of the three assays are shown. The percentage of living, necrotic, early and late apoptotic cells are presented in each box of the graph. Graphs with quantitative data were based on the relative ratio of early, late, necrotic and live cells. The error bar indicates standard error. "P<0.05. (B) TUNEL assay results of U2OS and MG63 cells treated with MTZ $(0,0.5$ and $1 \mu \mathrm{M})$ for $2 \mathrm{~h}$. Nuclei were stained with DAPI and yellow cells were considered as apoptotic cells. Scale bar, $50 \mu \mathrm{m}$. MTZ, mitoxantrone; TUNEL, terminal deoxynucleotidyl-transferase-mediated dUTP nick end labeling.

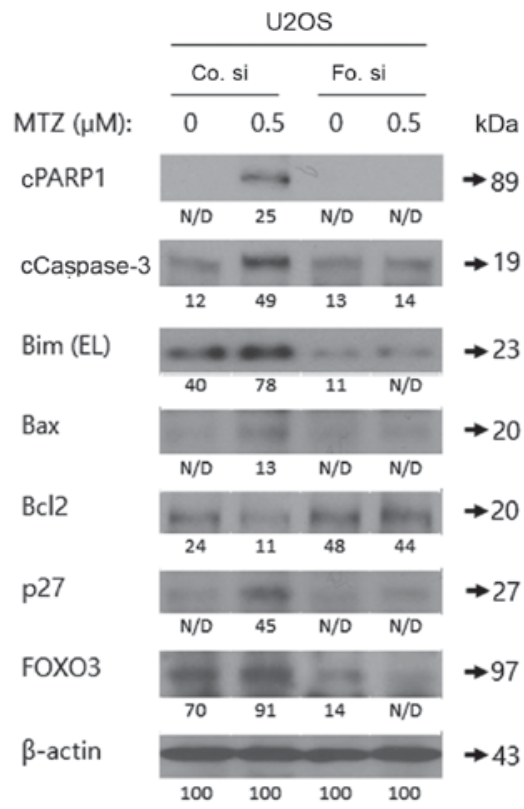

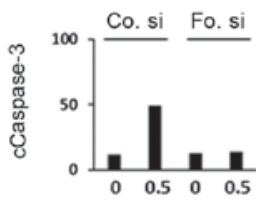
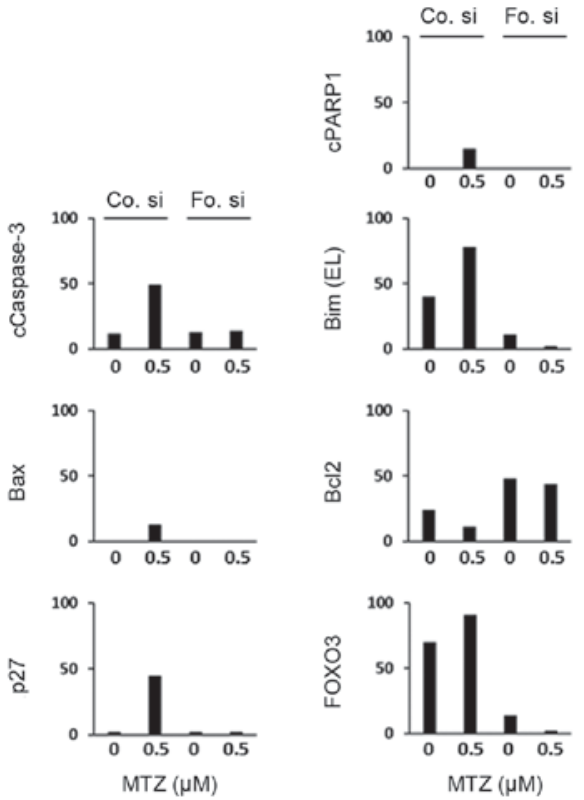

Figure 5. FOXO3 dependent-apoptosis in U2OS cells treated with MTZ. Western blotting results of U2OS cells transfected with control or FOXO3 siRNA and treated with MTZ ( 0 and $0.5 \mu \mathrm{M})$ for $48 \mathrm{~h}$, followed by blotting with specific antibodies as indicated. Graphs with quantitative data were based on the relative ratio of the indicated protein to $\beta$-actin. MTZ, mitoxantrone; EL, extra-large; FOXO3, forkhead-box O3; PARP1, poly(ADP-ribose) polymerase; c, cleaved; Co. si, control siRNA; Fo. si, FOXO3 siRNA. 
strongest activity, decreasing pS318/321 FOXO3 by $>50 \%$, compared with the DMSO vehicle control in U2OS cells (data not shown). Recently, Tarrado-Castellarnau et al (49) reported that methylseleninic acid was a selenium supplement that may have anti-cancer activity through the translocation of FOXO3 from the cytoplasm into the nucleus following the inhibition of Akt phosphorylation in U2OS cells stably expressing FOXO3; however, the endogenous FOXO3 expression levels or subcellular localization by methylseleninic acid in U2OS cells were not examined. In the present study, it was confirmed that MTZ induces nuclear localization and activation of FOXO3 in osteosarcoma cells. In addition, Guzmán-Pérez et al (50) demonstrated that benzylglucosinolate-derived isothiocyanate from tropaeolum majus promoted the translocation of FOXO1 from the cytoplasm into the nucleus following inhibition of Akt phosphorylation in U2OS cells stably expressing FOXO1. FOXO1 and FOXO3 are members of the human FOX gene family, which includes the distinct forkhead DNA-binding domain (6). Thus, they are known to have very similar cellular functions, including cell cycle arrest and cell death, as tumor-suppressive transcriptional factors (49). Indeed, there have been several previous reports indicating that FOXO1 has anti-tumor activity in osteosarcoma (50-52); therefore, it would be beneficial to study FOXO1 expression levels in osteosarcoma cells with MTZ.

Akt has numerous substrates that are phosphorylated, leading to activation or inhibition in cancer cells $(7,53)$. Previous research has investigated the effect of targeted therapies to treat various cancer types (53). In the present study, it was demonstrated that MTZ significantly inhibits pS473 Akt phosphorylation and causes nuclear localization and activation of FOXO3 in osteosarcoma cells (Fig. 2A and B). MTZ treatment inhibits proliferation of osteosarcoma cells in vitro, whereas silencing FOXO3 potently attenuates MTZ-mediated apoptosis in osteosarcoma cells. Similar to the present study, Yuan et al (54) evaluated the anti-cancer activity of MTZ analogs in Huh-7 human hepatoma cells and demonstrated that this MTZ analog inhibited the phosphorylation of Akt. Hu et al (55) reported that combination administration of MTZ and prednisolone, a steroid medication, may be one option for tumor therapy. They indicated that this combination displayed synergistic anti-cancer activity through inhibition of the Akt pathway, including phosphorylated glycogen synthase kinase $3 \beta$, ps6 ribosomal protein and pAMPK (55). Future studies will elucidate the molecular mechanism that MTZ treatment in osteosarcoma cells inhibits the phosphorylation of Akt, by investigating the upstream signaling proteins of Akt.

The aim was to demonstrate that MTZ displays anti-cancer activity through the regulation of the $\mathrm{Akt} / \mathrm{FOXO} 3$ pathway independent of p53 status, using osteosarcoma cell lines. Although p53 serves a key role in cell cycle arrest and apoptosis in numerous cancer types $(12,54,56,57)$, induction of cell cycle arrest and apoptosis in cancer cells by FOXO3 activation does not depend on p53 expression or status. For example, Matsuda et al (56) reported that treatment with OTS167, a maternal embryonic leucine zipper kinase (MELK) inhibitor, induced the upregulation of $\mathrm{p} 21$ protein expression in HCT116-p53(-/-) p53-null cells. They indicated that FOXO1 and FOXO3, two known tumor-suppressive Forkhead type transcriptional factors, may regulate p21 expression via activation following de-phosphorylation through treatment with a MELK inhibitor, independent of p53 status. Furthermore, Zhang et al (58) demonstrated that a tetrandrine derivative may potentially exert anti-cancer activity via induction of PUMA with a p53-independent mechanism. In that study, anti-cancer activity of tetrandrine derivatives were observed in wild-type and p53-null cancer cells, and inhibition of Akt/FOXO3 signaling may enhance tetrandrine derivative-mediated PUMA expression; therefore, consistent with previous reports, it was demonstrated that MTZ induces apoptosis in U2OS (p53 wild) and MG63 (p53 null) osteosarcoma cell lines and have identified a novel mechanism that MTZ regulates Akt/FOXO3, leading to the upregulation of p27, Bax and Bim and downregulation of Bcl-2. Further studies should knockdown p53 expression in U2OS cells and perform the same experiments, including confocal analysis, western blotting analysis and cell viability assay, of the present study to determine if the MTZ treatment has the same anti-cancer activity as p53 wild type U2OS cells.

To the best of our knowledge, this is the first report demonstrating that regulation of the $\mathrm{Akt} / \mathrm{FOXO} 3$ signaling pathway though treatment with MTZ is involved in controlling osteosarcoma cell development. Future studies should perform TMA analysis of patients with osteosarcoma, which will support the results of the present study and provide the clinical basis for a novel anti-osteosarcoma therapeutic strategy. Taken together, these results indicated that the anti-cancer activity of MTZ treatment may induce FOXO3-mediated apoptotic signaling pathways, and its application may be a novel chemotherapeutic agent for osteosarcoma.

\section{Acknowledgements}

Not applicable.

\section{Funding}

This research was supported by the Basic Science Research Program through the National Research Foundation of Korea NRF) funded by the Ministry of Education, Science and Technology (NRF-2014R1A6A3A04054307 and 2015R1A2A2A01003762).

\section{Availability of data and materials}

All data generated or analyzed during this study are included in this published article.

\section{Author's contributions}

SHP, JL, MAK, KYJ and JRK conceived and designed the experiments, Performed the experiments, analyzed the data, wrote the paper. KYJ and JRK take responsibility for the integrity of the data analysis. All authors reviewed the final manuscript.

\section{Ethics approval and consent to participate}

Not applicable. 


\section{Consent for publication}

Not applicable.

\section{Competing interests}

The authors declare that they have no competing interests.

\section{References}

1. Moore DD and Luu HH: Osteosarcoma. Cancer Treat Res 162 65-92, 2014

2. Lamoureux F, Trichet V, Chipoy C, Blanchard F, Gouin F and Redini F: Recent advances in the management of osteosarcoma and forthcoming therapeutic strategies. Expert Rev Anticancer Ther 7: 169-181,2007.

3. Luetke A, Meyers PA, Lewis I and Juergens H: Osteosarcoma treatment-where do we stand? A state of the art review. Cancer Treat Rev 40: 523-532, 2014.

4. Sakamoto A and Iwamoto Y: Current status and perspectives regarding the treatment of osteo-sarcoma: Chemotherapy. Rev Recent Clin Trials 3: 228-231, 2008.

5. Chou AJ and Gorlick R: Chemotherapy resistance in osteosarcoma: Current challenges and future directions. Expert Rev Anticancer Ther 6: 1075-1085, 2006.

6. Katoh M and Katoh M: Human FOX gene family (Review). Int J Oncol 25: 1495-1500, 2004

7. Alvarez B, Martinez-A C, Burgering BM and Carrera AC: Forkhead transcription factors contribute to execution of the mitotic programme in mammals. Nature 413: 744-747, 2001.

8. Furukawa-Hibi Y, Kobayashi Y, Chen C and Motoyama N FOXO transcription factors in cell-cycle regulation and the response to oxidative stress. Antioxid Redox Signal 7: 752-760, 2005.

9. Fu Z and Tindall DJ: FOXOs, cancer and regulation of apoptosis. Oncogene 27: 2312-2319, 2008.

10. Brunet A, Bonni A, Zigmond MJ, Lin MZ, Juo P, Hu LS, Anderson MJ, Arden KC, Blenis J and Greenberg ME: Akt promotes cell survival by phosphorylating and inhibiting a forkhead transcription factor. Cell 96: 857-868, 1999.

11. Park SH, Chung YM, Ma J, Yang Q, Berek JS and Hu MC: Pharmacological activation of $\mathrm{FOXO} 3$ suppresses triple-negative breast cancer in vitro and in vivo. Oncotarget 7: 42110-42125, 2016.

12. Park SH, Lee JH, Berek JS and Hu MC: Auranofin displays anticancer activity against ovarian cancer cells through FOXO3 activation independent of p53. Int J Oncol 45: 1691-1698, 2014.

13. Fei M, Zhao Y, Wang Y, Lu M, Cheng C, Huang X, Zhang D, $\mathrm{Lu}$ J, He S and Shen A: Low expression of Foxo3a is associated with poor prognosis in ovarian cancer patients. Cancer Invest 27: $52-59,2009$.

14. Lu M, Zhao Y, Xu F, Wang Y, Xiang J and Chen D: The expression and prognosis of FOXO3a and Skp2 in human ovarian cancer. Med Oncol 29: 3409-3415, 2012.

15. Jancic J, Nikolic B, Ivancevic N, Djuric V, Zaletel I, Stevanovic D, Peric S, van den Anker JN and Samardzic J: Multiple sclerosis in pediatrics: Current concepts and treatment options. Neurol Ther 5: 131-143, 2016.

16. Atwal M, Lishman EL, Austin CA and Cowell IG: Myeloperoxidase enhances etoposide and mitoxantrone-mediated DNA damage: A target for myeloprotection in cancer chemotherapy. Mol Pharmacol 91: 49-57, 2017.

17. Casadei B, Pellegrini C, Pulsoni A, Annechini G, De Renzo A, Stefoni V, Broccoli A, Gandolfi L, Quirini F, Tonialini L, et al: 90-yttrium-ibritumomab tiuxetan consolidation of fludarabine, mitoxantrone, rituximab in intermediate/high-risk follicular lymphoma: Updated long-term results after a median follow-up of 7 years. Cancer Med 5: 1093-1097, 2016.

18. Nussbaum N, George DJ, Abernethy AP, Dolan CM, Oestreicher N, Flanders S and Dorff TB: Patient experience in the treatment of metastatic castration-resistant prostate cancer: State of the science. Prostate Cancer Prostatic Dis 19: 111-121, 2016.

19. Hasinoff BB, Wu X, Patel D, Kanagasabai R, Karmahapatra S and Yalowich JC: Mechanisms of action and reduced cardiotoxicity of pixantrone; a topoisomerase II targeting agent with cellular selectivity for the topoisomerase II $\alpha$ isoform. J Pharmacol Exp Ther 356: 397-409, 2016.
20. Ferrer A, Marce S, Bellosillo B, Marcé S, Bellosillo B, Villamor N, Bosch F, López-Guillermo A, Espinet B, Solé F, Montserrat E, Campo E and Colomer D: Activation of mitochondrial apoptotic pathway in mantle cell lymphoma: High sensitivity to mitoxantrone in cases with functional DNA-damage response genes. Oncogene 23: 8941-8949, 2004.

21. Chong CR and Sullivan DJ Jr: New uses for old drugs. Nature 448 : 645-646, 2007.

22. Rena G, Woods YL, Prescott AR, Peggie M, Unterman TG, Williams MR and Cohen P: Two novel phosphorylation sites on FKHR that are critical for its nuclear exclusion. EMBO J 21: 2263-2271, 2002.

23. Bae JS, Lee J, Park Y, Park K, Kim JR, Cho DH, Jang KY and Park SH: Attenuation of MUC4 potentiates the anticancer activity of auranofin via regulation of the Her $2 / \mathrm{Akt} / \mathrm{FOXO} 3$ pathway in ovarian cancer cells. Oncol Rep 38: 2417-2425, 2017.

24. ESMO/European Sarcoma Network Working Group: Bone sarcomas: ESMO clinical practice guidelines for diagnosis, treatment and follow-up. Ann Oncol 25: iii113-iii123, 2014

25. Zambo I and Veselý K: WHO classification of tumours of soft tissue and bone 2013: The main changes compared to the 3rd edition. Cesk Patol 50: 64-70, 2014 (In Czech).

26. Schajowicz F, McGuire MH, Santini Araujo E, Muscolo DL and Gitelis S: Osteosarcomas arising on the surfaces of long bones. J Bone Joint Surg Am 70: 555-564, 1988.

27. Grimer RJ, Bielack S, Flege S, Cannon SR, Foleras G, Andreeff I, Sokolov T, Taminiau A, Dominkus M, San-Julian M, et al: Periosteal osteosarcoma-a European review of outcome. Eur J Cancer 41: 2806-2811, 2005.

28. Cesari M, Alberghini M, Vanel D, Palmerini E, Staals EL, Longhi A, Abate M, Ferrari C, Balladelli A and Ferrari S: Periosteal osteosarcoma: A single-institution experience. Cancer 117: 1731-1735, 2011.

29. Bernthal NM, Federman N, Eilber FR, Nelson SD, Eckardt JJ, Eilber FC and Tap WD: Long-term results ( $>25$ years) of a randomized, prospective clinical trial evaluating chemotherapy in patients with high-grade, operable osteosarcoma. Cancer 118: 5888-5893, 2012

30. Bielack SS, Smeland S, Whelan JS, Marina N, Jovic G, Hook JM, Krailo MD, Gebhardt M, Pápai Z, Meyer J, et al: Methotrexate, doxorubicin, and cisplatin (MAP) plus maintenance pegylated interferon Alfa-2b versus MAP alone in patients with resectable high-grade osteosarcoma and good histologic response to preoperative MAP: First results of the EURAMOS-1 good response randomized controlled trial. J Clin Oncol 33: 2279-2287, 2015.

31. Benjamin RS, Wagner MJ, Livingston JA, Ravi V and Patel SR: Chemotherapy for bone sarcomas in adults: The MD anderson experience. Am Soc Clin Oncol Educ Book: e656-e660, 2015.

32. Su W, Lai Z, Wu F, Lin Y, Mo Y, Yang Z and Wu J: Clinical efficacy of preoperative chemotherapy with or without ifosfamide in patients with osteosarcoma of the extremity: Meta-analysis of randomized controlled trials. Med Oncol 32: 481, 2015.

33. Navid F, Willert JR, McCarville MB, Furman W, Watkins A Roberts W and Daw NC: Combination of gemcitabine and docetaxel in the treatment of children and young adults with refractory bone sarcoma. Cancer 113: 419-425, 2008.

34. Rodriguez-Galindo C, Daw NC, Kaste SC, Meyer WH, Dome JS, Pappo AS, Rao BN and Pratt CB: Treatment of refractory osteosarcoma with fractionated cyclophosphamide and etoposide. J Pediatr Hematol Oncol 24: 250-255, 2002.

35. Saylors RL 3rd, Stine KC, Sullivan J, Kepner JL, Wall DA, Bernstein ML, Harris MB, Hayashi R and Vietti TJ; Pediatric Oncology Group: Cyclophosphamide plus topotecan in children with recurrent or refractory solid tumors: A pediatric oncology group phase II study. J Clin Oncol 19: 3463-3469, 2001.

36. Merimsky O, Meller I, Kollender Y, Issakov J, Flusser G and Inbar M: Gemcitabine in bone sarcoma resistant to doxorubicin-based chemotherapy. Sarcoma 4: 7-10, 2000.

37. Goorin AM, Harris MB, Bernstein M, Ferguson W, Devidas M, Siegal GP, Gebhardt MC, Schwartz CL, Link M and Grier HE: Phase II/III trial of etoposide and high-dose ifosfamide in newly diagnosed metastatic osteosarcoma: A pediatric oncology group trial. J Clin Oncol 20: 426-433, 2002.

38. Van Winkle P, Angiolillo A, Krailo M, Cheung YK, Anderson B, Davenport V, Reaman G and Cairo MS: Ifosfamide, carboplatin, and etoposide (ICE) reinduction chemotherapy in a large cohort of children and adolescents with recurrent/refractory sarcoma: The children's cancer group (CCG) experience. Pediatr Blood Cancer 44: 338-347, 2005. 
39. Michelagnoli MP, Lewis IJ, Gattamaneni HR, Bailey CC and Lashford LS: Ifosfamide/etoposide alternating with high-dose methotrexate: Evaluation of a chemotherapy regimen for poor-risk osteosarcoma. Br J Cancer 79: 1174-1178, 1999.

40. Anderson PM, Wiseman GA, Dispenzieri A, Arndt CA, Hartmann LC, Smithson WA, Mullan BP and Bruland OS: High-dose samarium-153 ethylene diamine tetramethylene phosphonate: Low toxicity of skeletal irradiation in patients with osteosarcoma and bone metastases. J Clin Oncol 20: 189-196, 2002

41. Hattinger CM, Vella S, Tavanti E, Fanelli M, Picci P and Serra M: Pharmacogenomics of second-line drugs used for treatment of unresponsive or relapsed osteosarcoma patients. Pharmacogenomics 17: 2097-2114, 2016.

42. Liu T,Li Z,Zhang Q, De Amorim Bernstein K, Lozano-Calderon S, Choy E, Hornicek FJ and Duan Z: Targeting ABCB1 (MDR1) in multi-drug resistant osteosarcoma cells using the CRISPR-Cas9 system to reverse drug resistance. Oncotarget 7: 83502-83513, 2016.

43. Buondonno I, Gazzano E, Jean SR, Audrito V, Kopecka J, Fanelli M, Salaroglio IC, Costamagna C, Roato I, Mungo E, et al: Mitochondria-targeted doxorubicin: A new therapeutic strategy against doxorubicin-resistant osteosarcoma. Mol Cancer Ther 15 2640-2652, 2016.

44. Chen Z, Guo J, Zhang K and Guo Y: TP53 mutations and survival in osteosarcoma patients: A meta-analysis of published data. Dis Markers 2016: 4639575, 2016.

45. Ru JY, Cong Y,Kang WB, Yu L, Guo T and Zhao JN: Polymorphisms in TP53 are associated with risk and survival of osteosarcoma in a Chinese population. Int J Clin Exp Pathol 8: 3198-3203, 2015.

46. Yao D, Cai GH, Chen J, Ling R, Wu SX and Li YP: Prognostic value of $\mathrm{p} 53$ alterations in human osteosarcoma: A meta analysis. Int J Clin Exp Pathol 7: 6725-6733, 2014.

47. Pakos EE, Kyzas PA and Ioannidis JP: Prognostic significance of TP53 tumor suppressor gene expression and mutations in human osteosarcoma: A meta-analysis. Clin Cancer Res 10: 6208-6214, 2004.

48. Ozaki T, Wu D, Sugimoto H, Nagase $H$ and Nakagawara A: Runt-related transcription factor 2 (RUNX2) inhibits p53-dependent apoptosis through the collaboration with HDAC6 in response to DNA damage. Cell Death Dis 4: e610, 2013.

49. Tarrado-Castellarnau M, Cortes R, Zanuy M, TarragóCelada J, Polat IH, Hill R, Fan TW, Link W and Cascante M: Methylseleninic acid promotes antitumour effects via nuclear FOXO3a translocation through Akt inhibition. Pharmacol Res 102: 218-234, 2015
50. Guzman-Perez V, Bumke-Vogt C, Schreiner M, Mewis I, Borchert A and Pfeiffer AF: Benzylglucosinolate Derived Isothiocyanate from Tropaeolum majus Reduces Gluconeogenic Gene and Protein Expression in Human Cells. PLoS One 11: e0162397, 2016.

51. Jin S, Pang RP, Shen JN, Huang G, Wang J and Zhou JG: Grifolin induces apoptosis via inhibition of PI3K/AKT signalling pathway in human osteosarcoma cells. Apoptosis 12: 1317-1326, 2007.

52. Pei H, Jin Z, Chen S, Sun X, Yu J and Guo W: MiR-135b promotes proliferation and invasion of osteosarcoma cells via targeting FOXO1. Mol Cell Biochem 400: 245-252, 2015.

53. Toker A and Marmiroli S: Signaling specificity in the Akt pathway in biology and disease. Adv Biol Regul 55: 28-38, 2014.

54. Yuan CL, Lin SW and Cheng MH: Inhibition of Molecular Signaling in Huh-7 Cells by AM3: A Novel Chemotherapeutic Agent for Hepatocellular Carcinoma. Med Chem: 49-56,2016.

55. Hu T, Cao H, Yang C, Zhang L, Jiang X, Gao X, Yang F, He G, Song X, Tong A, et al: LHD-modified mechanism-based liposome coencapsulation of Mitoxantrone and Prednisolone using novel lipid bilayer fusion for tissue-specific colocalization and synergistic antitumor effects. ACS App Mater Interfaces 8: 6586-6601, 2016.

56. Matsuda T, Kato T, Kiyotani K, Tarhan YE, Saloura V, Chung S, Ueda K, Nakamura Y and Park JH: p53-independent p21 induction by MELK inhibition. Oncotarget 8: 57938-57947, 2017.

57. Liu $\mathrm{H}$, Yin J, Wang $\mathrm{C}, \mathrm{Gu} \mathrm{Y}$, Deng $\mathrm{M}$ and $\mathrm{He} \mathrm{Z}$ : FOXO3a mediates the cytotoxic effects of cisplatin in lung cancer cells. Anticancer Drugs 25: 898-907, 2014.

58. Zhang YX, Liu XM, Wang J, Li J, Liu Y, Zhang H, Yu XW and Wei N: Inhibition of AKT/FoxO3a signaling induced PUMA expression in response to p53-independent cytotoxic effects of H1: A derivative of tetrandrine. Cancer Biol Ther 16: 965-975, 2015. 